\author{
Ralph Berther \\ Michael A. Patak \\ Boris Eckhardt \\ Sukru M. Erturk \\ Christoph L. Zollikofer
}

\section{Comparison of neutral oral contrast versus positive oral contrast medium in abdominal multidetector CT}

Received: 24 October 2007

Revised: 8 February 2008

Accepted: 23 February 2008

Published online: 15 April 2008

(C) European Society of Radiology 2008

R. Berther - M. A. Patak - B. Eckhardt C. L. Zollikofer

Institute of Radiology, Kantonsspital, Winterthur, Switzerland

M. A. Patak $(\bowtie)$

Institute of Diagnostic, Interventional and Paediatric Radiology, Inselspital, University Hospital of Bern,

3010 Bern, Switzerland

e-mail: michael.patak@insel.ch

Tel.: +41-31-6322111

Fax: +41-31-6324874

S. M. Erturk

Department of Radiology, Sisli Etfal

Education and Research Hospital,

Istanbul, Turkey

\begin{abstract}
To determine whether neutral contrast agents with waterequivalent intraluminal attenuation can improve delineation of the bowel wall and increase overall image quality for a non-selected patient population, a neutral oral contrast agent (3\% mannitol) was administered to 100 patients referred for abdominal multidetector row computed tomography (MDCT). Their results were compared with those of 100 patients given a positive oral contrast agent. Qualitative and quantitative measurements were done on different levels of the gastrointestinal tract by three experienced readers. Patients given the neutral oral contrast agent showed significant better qualitative results for bowel distension $(P<0.001)$, homogeneity of the luminal content $(P<0.001)$, delineation of the bowel-
\end{abstract}

wall to the lumen $(P<0.001)$ and to the mesentery $(P<0.001)$ and artifacts $(P<0.001)$, leading to a significant better overall image quality $(P<$ 0.001 ) than patients receiving positive oral contrast medium. The quantitative measurements revealed significant better distension $(P<0.001)$ and wall to lumen delineation $(P<0.001)$ for the patients receiving neutral oral contrast medium. The present results show that the neutral oral contrast agent (mannitol) produced better distension, better homogeneity and better delineation of the bowel wall leading to a higher overall image quality than the positive oral contrast medium in a non-selected patient population.

Keywords Multidetector row CT . Oral contrast media .

Abdominal radiology $\cdot$ Clinical study

\section{Introduction}

Recent advances in multidetector row computed tomography (MDCT) have led to a paradigm shift in the diagnostic approach to abdominal imaging, especially of the vascular system and solid organs [1-3]. The ability of MDCT to acquire imaging data with near isotropic voxel size allows for high-quality reconstruction in any possible viewing plane. The new display methods help to better communicate the diagnostic information in a way that is more understandable to the referring physicians. However, they have also changed the way imaging of the gastrointestinal tract (GIT) is perceived. The standard GIT distension method for MDCT of the abdomen employs high-attenuation contrast agents [diluted iodine (DI) or barium suspension] to label mainly the stomach and small bowel. With the advent of the new techniques, the use of high-attenuation GIT distension material should to be re-evaluated since it may degrade the image quality in procedures requiring maximum intensity reconstruction for abdominal MDCT angiography [4]. More importantly, it may fail to provide adequate mural enhancement for diagnosis of GIT abnormalities due to the almost non-existent difference between the enhanced bowel wall and the high-attenuation intraluminal content. Ideally, an oral contrast medium should have a high distension capability that delivers optimal differentiation between lumen and bowel wall.

Neutral intraluminal contrast agents could produce improved image quality and facilitate the diagnosis of GIT wall abnormalities since their attenuation of the bowel 
lumen approaches that of water. Neutral agents have proved to be effective in the diagnosis of Crohn's disease [5-7], neoplasms [8], and bowel-ischemia [9]. They are also used to distend and delineate the stomach and duodenum in imaging of pancreatic and biliary disease $[10,11]$. The most widely used neutral contrast agent is water. It has excellent contrast behaviour in the upper GIT. Its clinical use for the distal parts of the small bowel, however, is limited due to its rapid absorption [10-15]. Additives can slow the absorption of water by increasing the osmolarity $[16,17]$. One such additive is mannitol, an inexpensive and easy-to-use neutral oral contrast agent. To the best of our knowledge, mannitol has never been tested for abdominal MDCT imaging in a large group of non-selected patient poulation, although it has been tested for selected patients with small bowel disease [16].

We compared the image quality of an orally administered neutral contrast agent ( $3 \%$ mannitol) with that of a positive oral contrast agent $(3 \% \mathrm{DI})$ in MDCT of the abdomen and pelvis.

\section{Materials and methods}

\section{Patients}

A total of 200 consecutive patients (101 male, 99 female) referred to our department between January 2004 and May 2004 for MDCT of the abdomen and pelvis with intravenous contrast enhancement were studied. The indications for MDCT in both groups did not differ statistically with regard to their distribution (chi-square test). The study was approved by our Institutional Review Board.

Patients were divided into two groups of 100 patients each. The patients were randomly selected by a predefined list to participate in either of the two groups. The neutral oral contrast group (50 men, 50 women; mean age: $62.6 \pm$ 13.7 years) was given a $3 \%$ mannitol preparation, the positive oral contrast group (51 men, 49 women; mean age: $60.6 \pm 17$ years) a $3 \%$ DI solution. There were no significant intergroup differences regarding gender distribution (chisquare test) or age (Student's $t$-test).

The indication for the MDCT examination was as follows: oncology 53\%/56\% (neutral contrast group/DI group respectively), emergency radiology $29 \%$ and $28 \%$ and surgical evaluation, either preoperatively or postoperatively $18 \%$ for both groups.

\section{Preparation of oral contrast agents}

Administration of the contrast agents was standardized. The patients in each group received $1,000 \mathrm{ml}$ of liquid contrast solution mixed shortly before use. All the patients were instructed to drink the whole amount regularly over a 60-min period while remaining in a supervised area in our department where all potential side effects and complaints could be monitored by the nursing staff, such as vomiting, abdominal pain, diarrhea and allergic reactions.

The positive oral contrast group was given a 3\% DI solution consisting of $30 \mathrm{ml}$ of megluminioxitalamat (Telebrix Gastro, Guerbet, France) diluted in $970 \mathrm{ml}$ of tap water according to our clinical routine. Megluminioxitalamat contains $300 \mathrm{mg} / \mathrm{ml}$ iodine according to our clinical routine.

The neutral oral contrast group received a solution of $3 \%$ mannitol, which consists of $30 \mathrm{~g}$ of mannit sugar dissolved in 1,000 $\mathrm{ml}$ of tap water. This solution is well known from its application in abdominal magnetic resonance imaging (MRI) as published in previous studies [18].

Before imaging an intravenous line was placed in all patients. The patients were placed head first and supine on the CT table. While on the table they received rectal contrast medium consisting of $500 \mathrm{ml} \mathrm{3 \%}$ DI according to our standard clinical protocol.

\section{Imaging}

All examinations were performed on a 16-detector row MDCT scanner (Brilliance, Philips Imaging System, The Netherlands). A power injector (Tomojet, Doltron, Switzerland) administered $120 \mathrm{ml}$ of intravenous contrast material at a concentration of $300 \mathrm{mg} / \mathrm{ml}$ iodine [Ultravist 300 (Bayer-Schering, Germany), Xenetix 300 (Guerbet, France) or Imagopaque 300 (GE, USA)] with an injection rate of $1.5-2.5 \mathrm{ml} / \mathrm{s}$. The administration of contrast was followed by a flush of $50 \mathrm{ml}$ normal saline at the same injection rate. After coronal scout images were obtained, intravenous contrast bolus was initiated, followed 60-90 s later by single phase acquisition from the xiphoid to the symphysis using a $16 \times 1.5-\mathrm{mm}$ detector configuration, creating $5-\mathrm{mm}$ transverse sections. Coronal reconstructions $3-\mathrm{mm}$ thick were automatically generated by the computer from the anterior abdominal wall to the paravertebral muscles (Fig. 1).

\section{Image analysis}

Three experienced radiologists (R.B., M.A.P., B.E.), each with more than 6 years experience in body imaging, independently reviewed the images from each examination in transverse and coronal planes on a picture archiving and communication system workstation (AGFA, Japan). Because high-attenuation contrast was being compared to neutral intraluminal contrast, it was impossible to blind the readers to the oral contrast agent administered. All reviewers were blinded to the clinical history.

An incremental five-point scale $(0=$ worst, $4=$ best $)$ was used to rate images from each examination for bowel 
Fig. 1 Coronal reformation images of abdominal MDCT after oral administration of neutral (mannitol) (a) or positive (megluminioxitalamat) contrast (b). Direct comparison shows a marked and constant distension of the lumen with uniform attenuation in the neutral contrast prepared patient (a), and pronounced and increasing luminal attenuation in the positive contrast prepared patient (b)
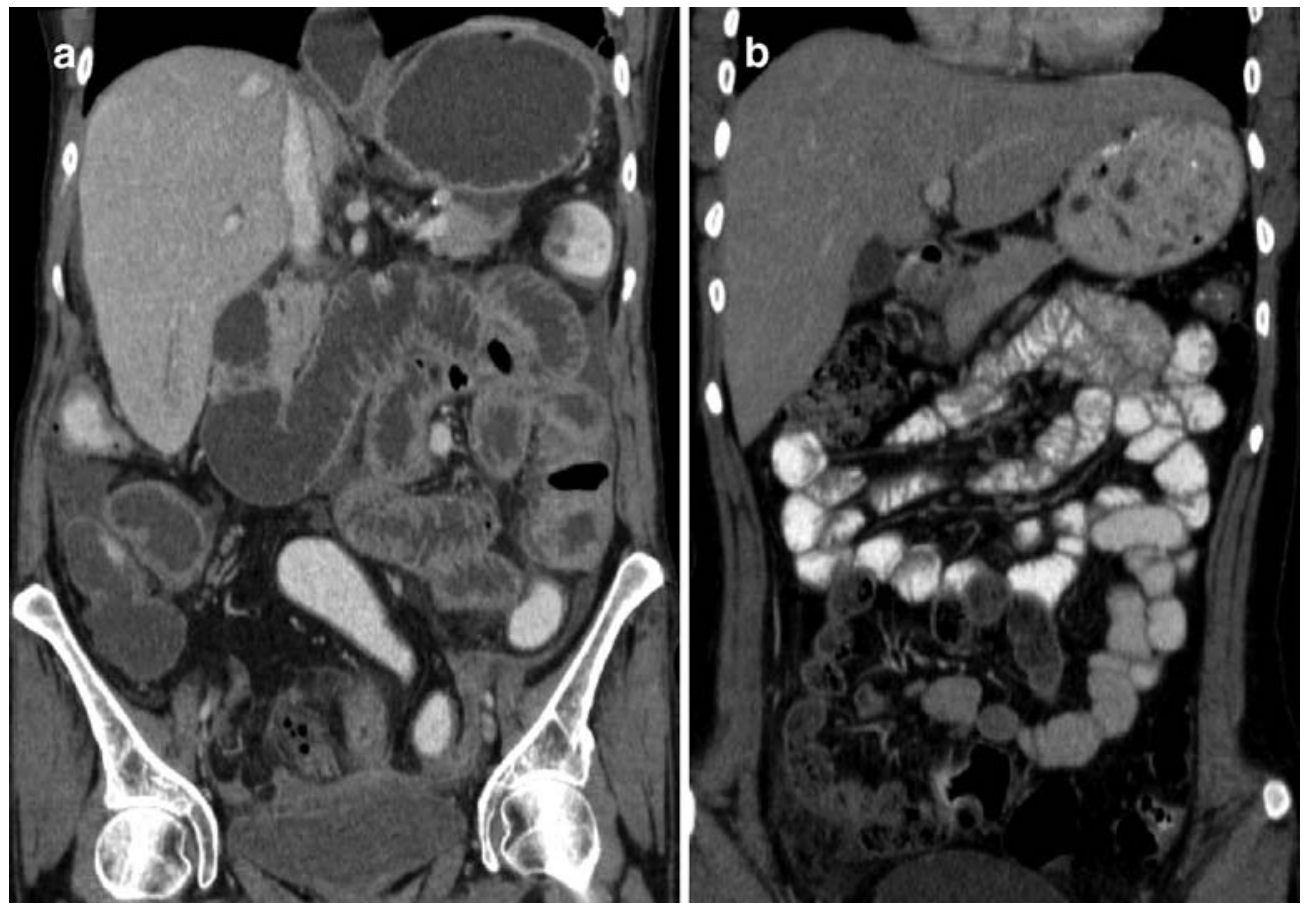

distension, homogeneity of the lumen, delineation of the bowel wall, presence of artifacts and overall image quality.

Overall bowel distention was graded from totally collapsed (0) to maximal distension (4); homogeneity of the lumen from massively inhomogeneous $(0)$ to completely homogeneous (4). The delineation of the bowel wall was rated twice from indiscernible $(0)$ to clearly visible (4), once against the intraluminal contrast and once against the surrounding extraintestinal tissue. The presence of artifacts ran from no artifacts (0) to serious image degradation due to artifacts (4). Finally, overall image quality was assessed from unreadable (0) to perfect (4).

To prove the aspects seen in the qualitative evaluation, additional quantitative measurements were performed by one of the researchers (R.B.) at least 2 weeks apart from the qualitative reading. The maximum cross-sectional diameter of the antrum of the stomach, horizontal part of the duodenum, proximal jejunum, and terminal ileum were measured perpendicular to the axis of the lumen using the outer margins of the intestinal wall for each patient. Attenuation [in Hounsfield units (HU)] of the lumen and gastrointestinal wall was measured at the same levels of the stomach, duodenum, jejunum, and terminal ileum. Attenuation of the lumen was measured by placing a region of interest (ROI) within a well-distended segment of the small bowel section. Attenuation of the wall was measured by first zooming into the image section until the wall was clearly visible, then placing an ROI over the bowel wall. The ROI had to have a minimal diameter of $2 \mathrm{~mm}$.

For each measured section of the GIT, the contrast for the bowel wall was calculated as the difference in attenuation values (contrast) of the lumen and wall by subtracting the HU value obtained from the lumen from the HU value obtained from the corresponding gastrointestinal wall.

\section{Statistical analysis}

For all readers, the Mann-Whitney $U$-test was used to perform statistical analysis of the differences in median scores between the neutral oral contrast and positive oral contrast groups regarding bowel distention, homogeneity of the lumen, differentiation of the bowel wall against luminal content and surrounding fat, the presence of artifacts and overall image quality. Differences were considered significant if $P<0.05$. Inter-observer agreement was evaluated using linear-weighted kappa statistics where a kappa statistic $>0.75$ was considered as excellent agreement, $0.4-0.75$ as fair to good agreement, and $<0.4$ as poor agreement [19]. Values near zero or less than zero reflected only chance agreement.

Regarding the quantitative analysis, the differences in maximum diameters, HU values for the GIT lumen, and contrast values between the neutral oral contrast and positive oral contrast groups were compared using Student's unpaired $t$-test. Differences were considered significant if $P<0.05$.

\section{Results}

All patients tolerated the administration of oral contrast well, there was no vomiting, diarrhea, abdominal pain nor 
allergic reactions to both oral contrast. There were no serious side effects. All patients drank the designated amount of fluid within the given time span. MDCT could be obtained without major side effects in both groups.

\section{Qualitative evaluation}

The images were of sufficient quality to be evaluated, for a total of 200 assessed MDCT scans. The individual qualitative results for all readers are summarized in Fig. 2. The inter-observer variability was fair to good with kappa values of $0.44-0.67$.

The distension of the entire small bowel, homogeneity of the applied contrast medium, detection of the internal and external borders of the bowel wall, and the rate of artifacts were judged to be significantly better $(P<0.001)$ for the neutral oral contrast images than the positive oral contrast images. Also, the overall quality was adjudged to be significantly better $(P<0.001)$ for the neutral oral contrast group than for the positive oral contrast group.

The inter-observer variability was moderate to good for all sites with a high significance $(P<0.001)$ for all sites.

\section{Quantitative measurement}

The findings on all measurements are summarized in Table 1. The mean diameter of the antrum of the stomach measured $6.7 \mathrm{~cm}$ for neutral oral contrast group and $6.2 \mathrm{~cm}$ for the positive oral contrast group, not a significant difference. For all other locations, however, the diameter was significantly wider for the neutral oral contrast group. Attenuation of the bowel wall showed no significant intergroup difference in $\mathrm{HU}$ measurements at all locations. As expected, attenuation of the lumen at the level of the

\section{Qualitative Analysis of MDCT Images}

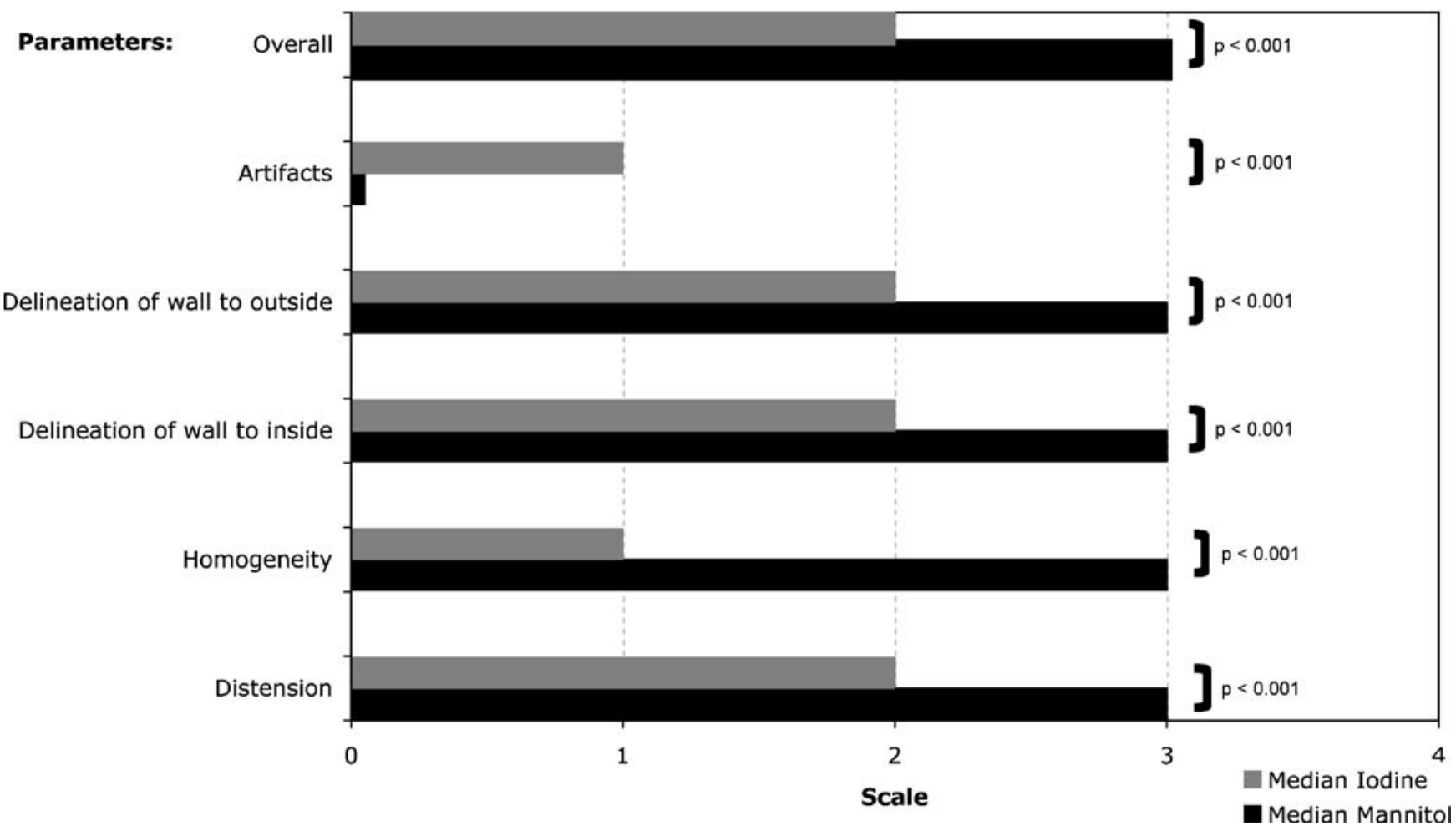

Fig. 2 Qualitative analysis of the MDCT images of 200 patients receiving either neutral (mannitol) $(n=100)$ or positive (DI) $(n=100)$ oral contrast medium. The images were evaluated for bowel distension, homogeneity of the lumen, delineation of the bowel wall, rate of artifacts, and overall image quality on axial as well as on coronal reconstructions by three experienced readers applying a five-point scale. Qualitative analysis of MDCT of 200 patients receiving either neutral (mannitol) $(\mathrm{n}=100)$ or positive (diluted iodine $(n=100)$ oral contrast medium. The images were evaluated for bowel distension, homogeneity of the lumen, delineation of the bowel wall, rate of artifacts, and overall image quality on axial as well as on coronal reconstructions by 3 experienced readers applying a 5-point scale. The median score for each reader for a given evaluation location is shown, with the range in parenthesis. For each reader, all qualitative assessments were significantly better for the neutral contrast (mannitol) group as indicated by the $p$ values using the unpaired Student's t-test 
Table 1 Quantitative measurements of MDCT images of 200 patients receiving either neutral (mannitol) $(n=100)$ or positive (DI) ( $n=100)$ oral contrast medium. The diameter and attenuation of the lumen and bowel wall were measured at different levels of the small bowel

\begin{tabular}{|c|c|c|c|c|c|}
\hline Location & Oral contrast & $\begin{array}{l}\text { Diameter } \\
(\mathrm{cm})\end{array}$ & $\begin{array}{l}\text { Attenuation of bowel wall } \\
(\mathrm{HU})\end{array}$ & $\begin{array}{l}\text { Attenuation of bowel lumen } \\
\text { (HU) }\end{array}$ & $\begin{array}{l}\text { Difference wall } \\
\text { to lumen }\end{array}$ \\
\hline \multirow[t]{3}{*}{ Stomach } & $\begin{array}{l}\text { Neutral contrast } \\
\text { (mannitol) }\end{array}$ & 6.7 & 69.3 & 2.3 & 67.0 \\
\hline & Positive contrast (DI) & 6.2 & 63.7 & 95.4 & -31.8 \\
\hline & & 0.35 & 0.05 & $<0.001$ & $<0.001$ \\
\hline \multirow[t]{3}{*}{ Duodenum } & $\begin{array}{l}\text { Neutral contrast } \\
\text { (mannitol) }\end{array}$ & 2.4 & 74.0 & 3.1 & 70.6 \\
\hline & Positive contrast (DI) & 1.9 & 67.9 & 86.3 & -18.5 \\
\hline & & $<0.001$ & 0.15 & $<0.001$ & $<0.001$ \\
\hline \multirow[t]{3}{*}{ Jejunum } & $\begin{array}{l}\text { Neutral contrast } \\
\text { (mannitol) }\end{array}$ & 2.1 & 67.1 & 6.8 & 60.3 \\
\hline & Positive contrast (DI) & 1.8 & 67.4 & 112.9 & -45.4 \\
\hline & & $<0.001$ & 0.85 & $<0.001$ & $<0.001$ \\
\hline \multirow[t]{3}{*}{$\begin{array}{l}\text { Terminal } \\
\text { ileum }\end{array}$} & $\begin{array}{l}\text { Neutral contrast } \\
\text { (mannitol) }\end{array}$ & 1.8 & 56.0 & 5.9 & 50.2 \\
\hline & Positive contrast (DI) & 1.5 & 60.3 & 205.4 & -145.1 \\
\hline & & $<0.001$ & 0.05 & $<0.001$ & $<0.001$ \\
\hline
\end{tabular}

stomach, duodenum, jejunum, and terminal ileum was significantly higher in the positive oral contrast group receiving DI.

A significantly higher difference between the bowel wall attenuation and the luminal content was calculated for the neutral contrast group compared to the positive oral contrast group $(P<0.001)$.

\section{Discussion}

The use of neutral oral contrast agents for CT enterography has been described by many authors [20-22]. These authors used neutral contrast agents specifically to investigate small bowel diseases, such as Cohn's disease, or for tumour staging. If the focus lay on other organs, they preferred positive intraluminal contrast to better distinguish small bowel structures from other organs, or intraluminal fluid from fluid collections outside the bowel.

There is rising interest in the use of neutral contrast agents for a wider range of indications or even routinely. In this context, a study of VoLumen (E-Z-EM, Lake Success, N.Y.), a commercially available, low attenuation contrast agent, reported good bowel distention and good visualization of the bowel wall [23]. Many aspects of mannitol resemble those of VoLumen. They are both definitely better than water for distension and conspicuity of the bowel wall. They also have a low side-effect profile. The main difference concerns the densities: due to its lower barium $(0.1 \%)$ content, VoLumen has slightly higher HU than mannitol (20-40 HU versus $0-10 \mathrm{HU})$. This can be helpful in depicting extraluminal pathologies, which often have the same attenuation as water.

With positive oral contrast agents, subtle changes in the bowel wall and its surrounding fat can be masked. The reasons for this are (1) the enhanced bowel wall may have the same attenuation as the positive contrast of the lumen and (2) because areas of high densities often occur due to heterogeneous intraluminal distribution, leading to artifacts with reduced visualization of the bowel wall and surrounding fat (Fig. 3).

The technical advances in MDCT technology, with high resolution image acquisition, multiplanar reconstruction, and computer workstations for anatomical reconstruction have made it possible to interpret every structure in abdominal CT studies, including organs, vessels, bones and also bowel, in a single examination (Fig. 4).

The ability of workstations to reconstruct anatomical details in every desirable plane has made it much easier to differentiate between the bowel and surrounding structures, as well as between intraluminal and extraluminal fluid. Orally administered neutral luminal contrast medium leads to good visualization of small bowel structures, clearly delineating lesions and even the normal bowel wall, the blood supply, and the status of the surrounding fat tissue [4, $5]$ without hampering the examination of other organs. The differentiation of extraluminal fluid could be difficult with neutral oral contrast medium. The above mentioned ability of multiplanar reconstruction compensates this drawback. Therefore neutral oral contrast agents, preferably those 


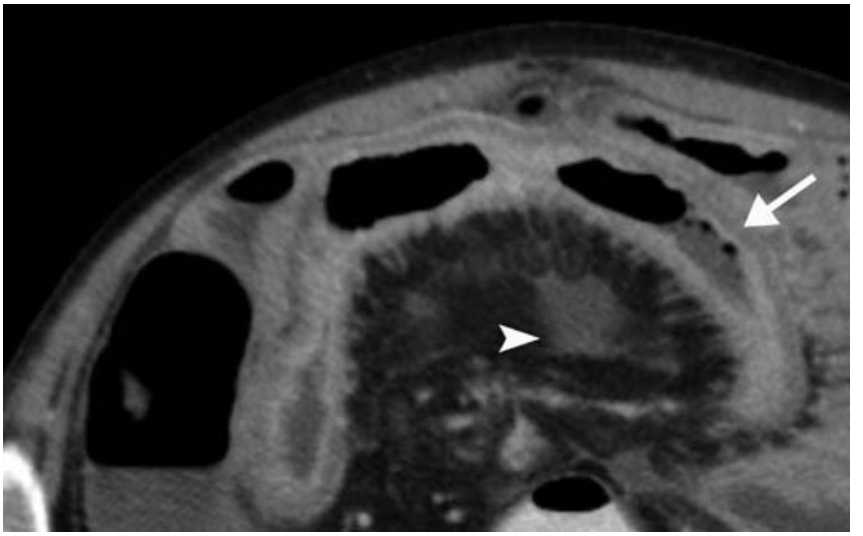

Fig. 3 Enlarged section of an axial plane of abdominal MDCT in a 57-year-old woman after oral preparation with neutral contrast medium (mannitol). The image shows Crohn's disease with intraabdominal abscesses (arrowhead), inflammation of the small bowel with markedly thickened wall (arrow). There is good contrast between intraluminal fluid and bowel wall. Note especially the strong mucosal enhancement

providing good distention of the bowel, merit routine application (Fig. 5).

Water as neutral contrast agent is quickly absorbed in the GIT, leading to insufficient bowel distention throughout [13]. We, therefore, used a $3 \%$ mannitol solution in the expectation that mannitol's slight osmotic effect would produce better distention. This contrast agent is used at some institutions as part of a routine MR protocol for the examination of the small bowel, providing good small bowel distension without absorption [8, 9, 14, 24].

One of the main goals of the present study was, therefore, to compare small bowel distention after oral administration of a 3\% mannitol-based contrast to that following oral administration of a positive 3\% DI-based contrast agent. For this purpose calculation of the whole small-bowel volume would be best, but no such technique is yet available. Quantitative measurement of the bowel diameter at different segments, considering the length of the small bowel, can only give an approximation of the overall distention. By choosing reproducible sites along the GIT at the level of the stomach (widest part), horizontal part of the duodenum (widest part), the proximal jejunum, and terminal ileum, we were able to compare our measurements.

We found significantly better distension of the small bowel with the neutral contrast (mannitol) than with the positive contrast (DI). The quantitative measurements were confirmed by the qualitative results by the three readers, with moderate to good inter-observer correlation. The diameter of the stomach did not differ significantly between the two contrast agent groups.

The results of the better distension can be explained by the higher osmotic effect of mannitol compared with DI. The water that is administered with the mannitol solution and the physiologic fluids secretion of the upper GI tract are retained by the high osmotic effect of the mannit sugar, which itself is non-digestible by the human body. On the other hand, DI has only a slight osmotic effect, therefore fluid is absorbed during the passage in the bowel and the distension is decreasing for the more distal bowel sections. The increase in attenuation of the bowel content can be explained by absorbtion of water and increasing concentration of iodine in the remaining water.

The quantitative analysis was performed by measuring the attenuation of the bowel wall and the attenuation of the fluid in the lumen at the locations mentioned above. Technical challenges occurred if the intraluminal fluid was too concentrated, thereby masking the bowel wall and leading to artifacts (Fig. 6). The difference in attenuation between the intraluminal fluid and enhanced bowel wall is the degree of bowel wall contrast. In our patients, the

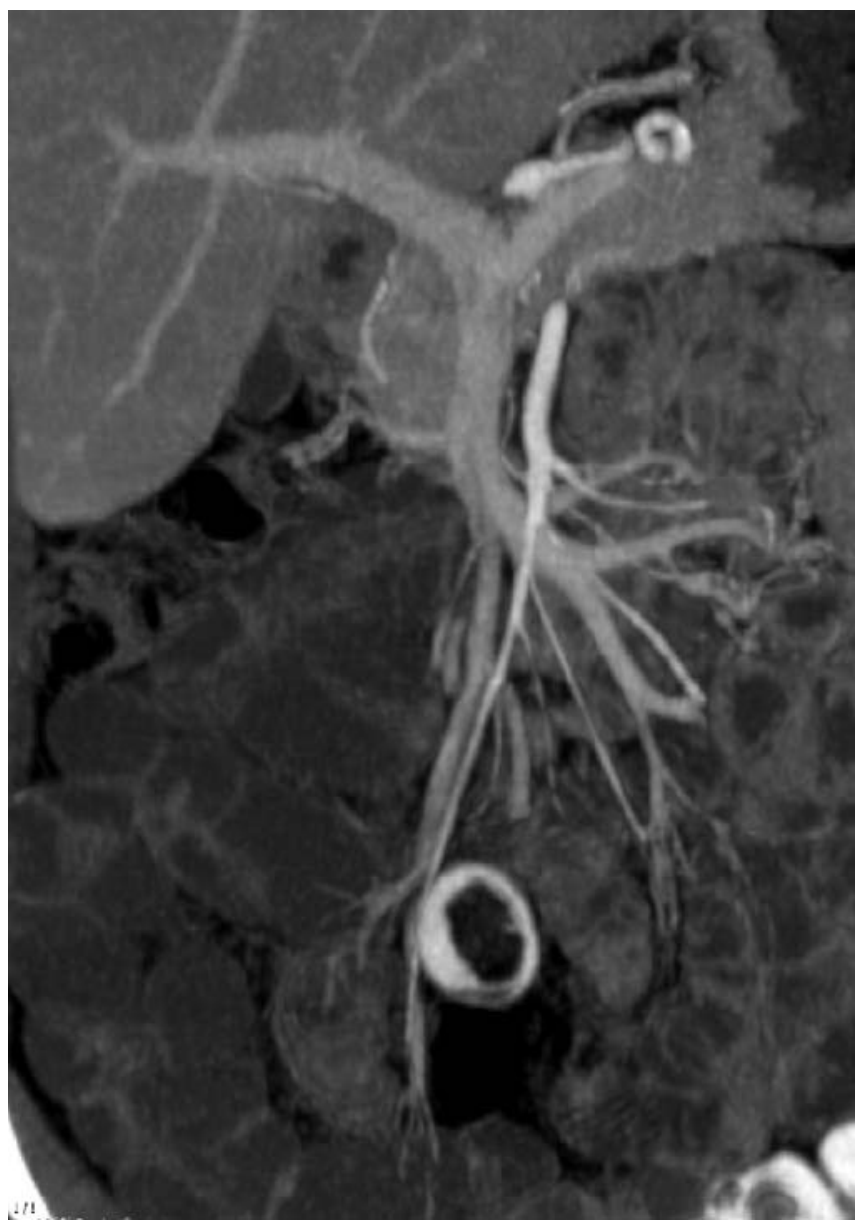

Fig. 4 Coronal maximum intensity projection (MIP) of abdominal MDCT in a 54-year-old woman after oral preparation with neutral contrast (mannitol). Note the well-extended small bowel. The neutral contrast medium in the small bowel allowed for easy MIP reconstruction, leading to better demonstration of the vessels than would have been the case with positive intraluminal contrast medium 


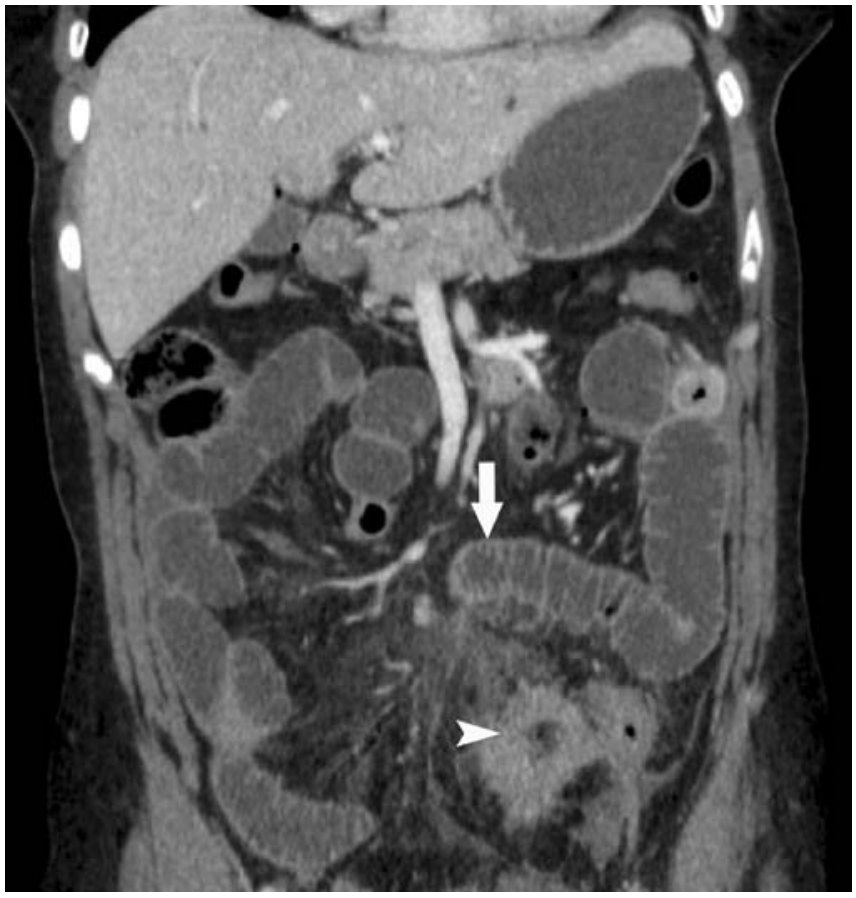

Fig. 5 Coronal plane of abdominal MDCT in a 37-year-old woman with diverticulitis presenting with circular thickening of the colonic wall and focal fatty infiltration (thickened colon displayed axial, arrowhead) following oral administration of neutral contrast medium (mannitol). The small bowel is well distended over its entire length with homogeneous attenuation of the luminal fluid. The small bowel wall is clearly differentiated, including the valvulae conniventes (arrow). There are no artifacts

neutral contrast agent led to very similar neutral fluid attenuation over the entire length of the small bowel, providing a homogeneous image. The neutral agent produced significantly better delineation of the enhanced bowel wall in the duodenum, jejunum and in the stomach, with contrast differences of 60-70 HU. Again, the three readers provided qualitative confirmation of the results with good inter-observer correlation.

For the terminal ileum the positive oral contrast group showed a high average contrast difference of $145 \mathrm{HU}$ between the luminal fluid versus the wall. Instead of the expected good visualization of the wall, however, artifacts caused by these high intraluminal contrast levels made evaluation of the wall in this segment difficult.

Even with the increasing luminal attenuation from the proximal to the distal small bowel in the neutral oral contrast medium group the oral contrast material application resulted in a homogeneous luminal contrast throughout to small bowel compared with the positive contrast. One reason for this may have been the inconsistent rate of oral uptake. Although the patients were told to drink the contrast agent regularly over a 60 -min period, they may have tended initially to drink too fast. However, since the effect was seen in almost all patients, osmotic dilution appears to have occurred as well.
Interestingly, there was a strong trend in the difference of the attenuations of the bowel wall at the level of the stomach and the terminal ileum. At the level of the stomach, there was a higher attenuation of the wall in the mannitol group compared wiyh the DI group, whereas it showed a higher attenuation at the terminal ileum within the DI group compared with the mannitol group. There is no clear explanation for this phenomenon since it is inconsistent for the different patient groups.

The overall image quality was judged to be slightly better in the neutral oral contrast group, with an undiminished ability to achieve a diagnosis.

\section{Conclusion}

The use of a neutral oral contrast agent led to good image quality with better distension of the small bowel and

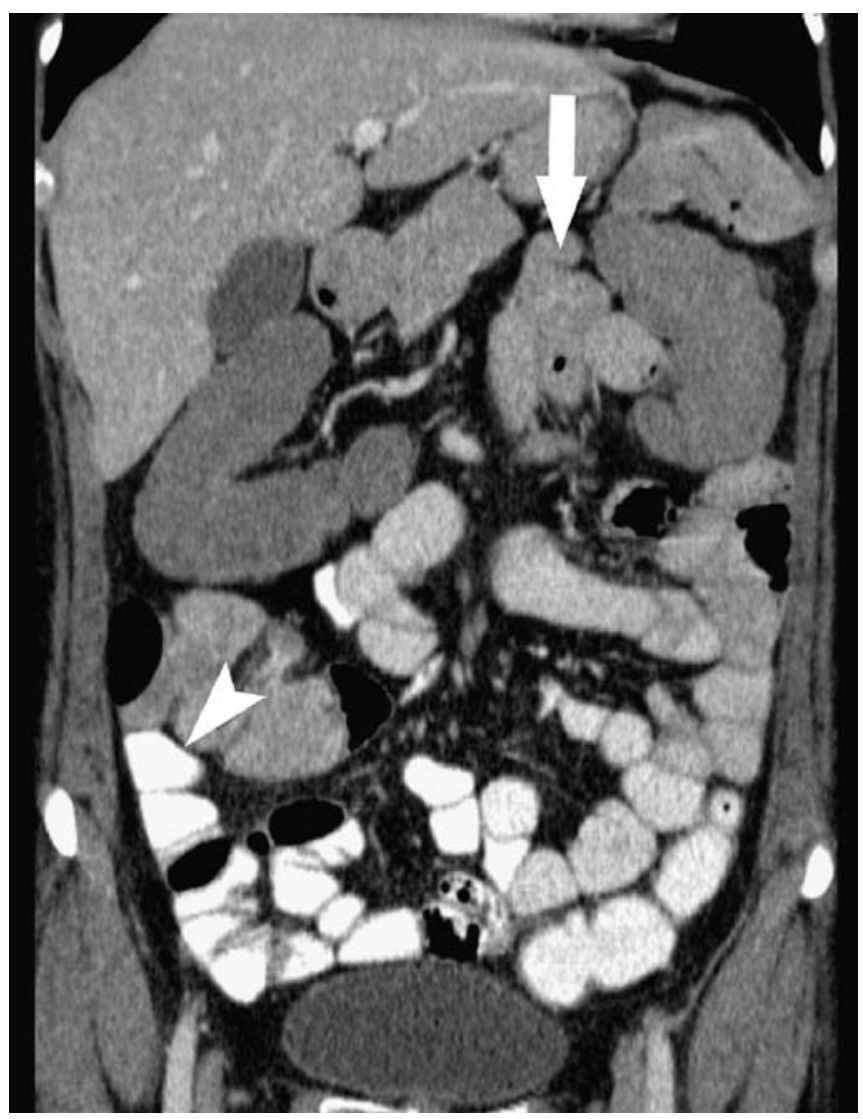

Fig. 6 Coronal plane of abdominal MDCT in a 54-year-old woman, prepared with orally administered positive contrast medium (Megluminioxitalamat, Telebrix Gastro, Guerbet, France). Heteroogeneous attenuation of the intraluminal fluid is evident, with increasing attenuation from the proximal small bowel (arrow) to the ileum (arrowhead). In the proximal small bowel, the bowel wall cannot be differentiated since the attenuation of the bowel wall matches that of the intraluminal fluid $(85 \mathrm{HU})$. High intraluminal attenuation (303 HU) hampered evaluation of the bowel wall in the distal part of the small bowel 
greater delineation of the bowel wall compared with the positive oral contrast agent. The neutral oral contrast did not mask the bowel wall as occurred with the positive oral contrast. Combined with state-of-the-art MDCT systems and workstations that allow reconstruction of lesions in every desired plane, neutral oral contrast agents can facilitate accurate diagnosis of lesions of the GIT. Based on the present results, they appear to be suited for the diagnosis in unselected patients.

\section{References}

1. Foley WD, Kerimoglu U (2004) Abdominal MDCT: liver, pancreas, and biliary tract. Semin Ultrasound CT MR 25:122-144

2. Napoli A, Fleischmann D, Chan FP et al (2004) Computed tomography angiography: state-of-the-art imaging using multidetector-row technology. J Comput Assist Tomogr 28(Suppl 1): S32-S45

3. Wintersperger BJ, Nikolaou K, Becker CR (2004) Multidetector-row CT angiography of the aorta and visceral arteries. Semin Ultrasound CT MR 25:2540

4. Horton KM, Fishman EK (2003) The current status of multidetector row CT and three-dimensional imaging of the small bowel. Radiol Clin North Am 41:199-212

5. Turetschek K, Schober E, Wunderbaldinger P et al (2002) Findings at helical CT-enteroclysis in symptomatic patients with crohn disease: correlation with endoscopic and surgical findings. J Comput Assist Tomogr 26:488-492

6. Wold PB, Fletcher JG, Johnson CD, Sandborn WJ (2003) Assessment of small bowel Crohn disease: noninvasive peroral CT enterography compared with other imaging methods and endoscopy - feasibility study. Radiology 229:275-281

7. Reittner P, Goritschnig T, Petritsch W et al (2002) Multiplanar spiral CT enterography in patients with Crohn's disease using a negative oral contrast material: initial results of a noninvasive imaging approach. Eur Radiol 12:2253-2257
8. Horton KM, Fishman EK (2004) Multidetector-row computed tomography and 3-dimensional computed tomography imaging of small bowel neoplasms: current concept in diagnosis. J Comput Assist Tomogr 28:106-116

9. Horton KM, Fishman EK (2001) Multidetector row CT of mesenteric ischemia: can it be done? Radiographics 21:1463-1473

10. Winter TC, Ager JD, Nghiem HV, Hill RS, Harrison SD, Freeny PC (1996) Upper gastrointestinal tract and abdomen: water as an orally administered contrast agent for helical CT. Radiology 201:365-370

11. Winter TC 3rd, Freeny PC, Nghiem HV (1996) Extrapancreatic gastrinoma localization: value of arterial-phase helical CT with water as an oral contrast agent. AJR Am J Roentgenol 166:5152

12. Horton KM, Eng J, Fishman EK (2000) Normal enhancement of the small bowel: evaluation with spiral CT. J Comput Assist Tomogr 24:67-71

13. Baert AL, Roex L, Marchal G, Hermans P, Dewilde D, Wilms G (1989) Computed tomography of the stomach with water as an oral contrast agent: technique and preliminary results. J Comput Assist Tomogr 13:633636

14. Gossios KJ, Tsianos EV, Demou LL et al (1991) Use of water or air as oral contrast media for computed tomographic study of the gastric wall: comparison of the two techniques. Gastrointest Radiol 16:293-297

15. Hori S, Tsuda K, Murayama S, Matsushita M, Yukawa K, Kozuka T (1992) CT of gastric carcinoma: preliminary results with a new scanning technique. Radiographics 12:257-268

16. Zhang LH, Zhang SZ, Hu HJ et al (2005) Multi-detector CT enterography with iso-osmotic mannitol as oral contrast for detecting small bowel disease. World J Gastroenterol 11:2324-2329
17. Antoch G, Kuehl H, Kanja J et al (2004) Dual-modality PET/CT scanning with negative oral contrast agent to avoid artifacts: introduction and evaluation. Radiology 230:879-885

18. Schunk K, Kersjes W, SchadmandFischer S, Grebe P, Kauczor HU, Thelen M (1995) [A mannitol solution as an oral contrast medium in pelvic MRT]. Rofo Fortschr Geb Rontgenstr Neuen Bildgeb Verfahr 163:60-66

19. Fleiss JL (1981) Statistical methods for rates and proportions. Wiley, New York

20. Bender GN, Maglinte DD, Kloppel VR, Timmons JH (1999) CT enteroclysis: a superfluous diagnostic procedure or valuable when investigating small-bowel disease? AJR Am J Roentgenol 172:373-378

21. Bender GN, Timmons JH, Williard WC, Carter J (1996) Computed tomographic enteroclysis: one methodology. Invest Radiol 31:43-49

22. Raptopoulos V (1989) Technical principles in CT evaluation of the gut. Radiol Clin North Am 27:631-651

23. Megibow AJ, Babb JS, Hecht EM et al (2006) Evaluation of bowel distention and bowel wall appearance by using neutral oral contrast agent for multidetector row CT. Radiology 238:87-95

24. Negaard A, Paulsen V, Sandvik L et al (2007) A prospective randomized comparison between two MRI studies of the small bowel in Crohn's disease, the oral contrast method and MR enteroclysis. Eur Radiol 17:2294-2301 